\title{
A NEW EFFICIENT PROCEDURE FOR THE ESTIMATION OF ONSET TIMES OF SEISMIC WAVES
}

\author{
Tetsuo Takanami* and Genshiro Kitagawa** \\ * Research Center for Earthquake Prediction, Faculty of Science, \\ Hokkaido University, Sapporo, Japan \\ ** The Institute of Statistical Mathematics, \\ Minato-ku, Tokyo, Japan
}

(Received October 22, 1987; Revised November 29, 1988)

\begin{abstract}
A computationally efficient procedure was developed for the fitting of a locally stationary autoregressive model. The amount of computations is bounded by a function of the data length and the model order only and does not depend on the number of possible arrival times. This facilitates automatic determination of arrival time by an on-line system. The on-line system FUNIMAR (fast univariate case of minimum AIC method of AR model fitting) was developed to implement the procedure:

The method was checked by applying it to weak seismic signals from earthquakes which were superimposed with background noises such as traffic noise, electronic hum noise, and heavy microtremor. Experimental results confirm the effectiveness of the method for determining the onset time of P-wave and suggest that an AR model of the fifth order is sufficient to determine the onset time of the wave even if it is buried in considerably heavy noises. It is also shown that the onset time of S-wave can also be successfully determined by the method.
\end{abstract}

\section{Introduction}

In recent years, the automatic processing of seismic signals for the detection of seismic activity has become realistic due to the establishment of a well-equipped nation-wide seismological network (HAMAGUCHI and SuZUKI, 1979).

In earthquake prediction it is necessary to rapidly distinguish a foreshock sequence from background seismicity of a region which is faciliated by rapid location of earthquakes and by processing large quantities of data.

Needless to say, the seismic signals observed by seismometers are contaminated by various kinds of signals, such as microtremors, microseisms, and artificial vibrations. These observed data sets have been traditionally handled by empirical methods based on the expertise of the human operator to single out real seismic signals from the various noises. For the automatic processing of seismic signals, it thus becomes necessary to develop a method that can automatically detect seismic wave from noisy data. 

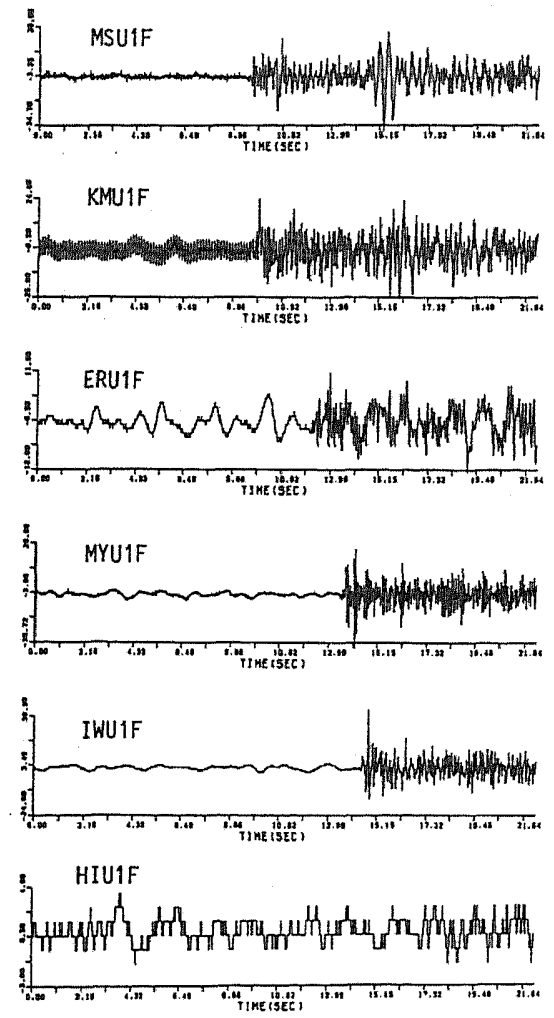

(a)
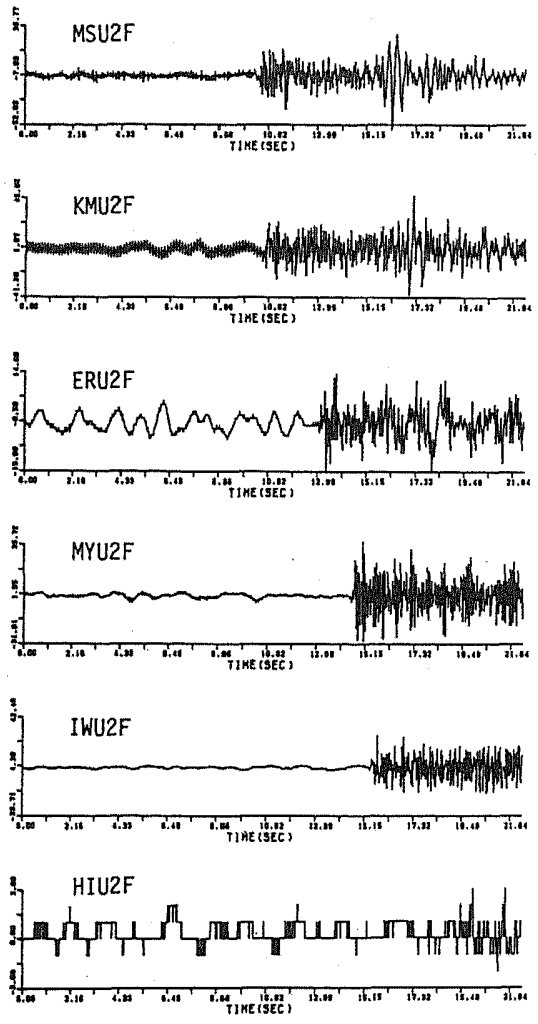

(b)

Fig. 1. Seismographs of up-down components recorded at the Hidaka network of RCEP and used in the present study. Their events are the foreshocks of the 1982 Urakawa-Oki Earthquake (off Urakawa). (a) Seismograms of foreshock No. 1. (b) Seismograms of foreshock No. 2.

Some attempts have been made based on the autoregressive (AR) modeling of the observed seismic signals (TJøstheim, 1975; ShIraI and Tokuhiro, 1979; Hamaguchi and Suzuki, 1979; Yokota et al., 1981; HamaguCHI and Morita, 1980; Morita and Hamaguchi, 1981; MAEda, 1985, 1986; Hasegawa et al., 1986). An AR model is very useful for the analysis of a stationary time series. However, from the statistical point of view, the main feature of the signals observed by seismometers is the non-stationary aspect. Although seismic waves are non-stationary, it might be reasonable to consider that it can be approximated by an AR model on each properly divided time interval (OZAKI and ToNG, 1975; KITAGAWA and AKAIKE, 1978). The use of the locally stationary AR model was thus considered desirable and it was shown that it is actually useful for detection of arrival of Pwaves in the noisy data (YoKota et al., 1981). A significant merit of the time series method is that we can automatically determine the arrival time of the P-waves by 

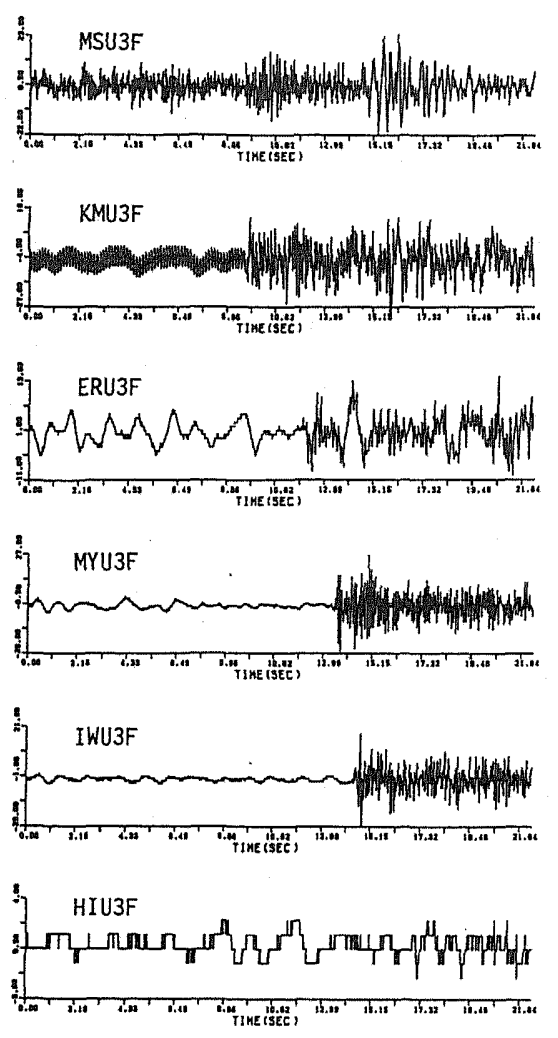

(c)
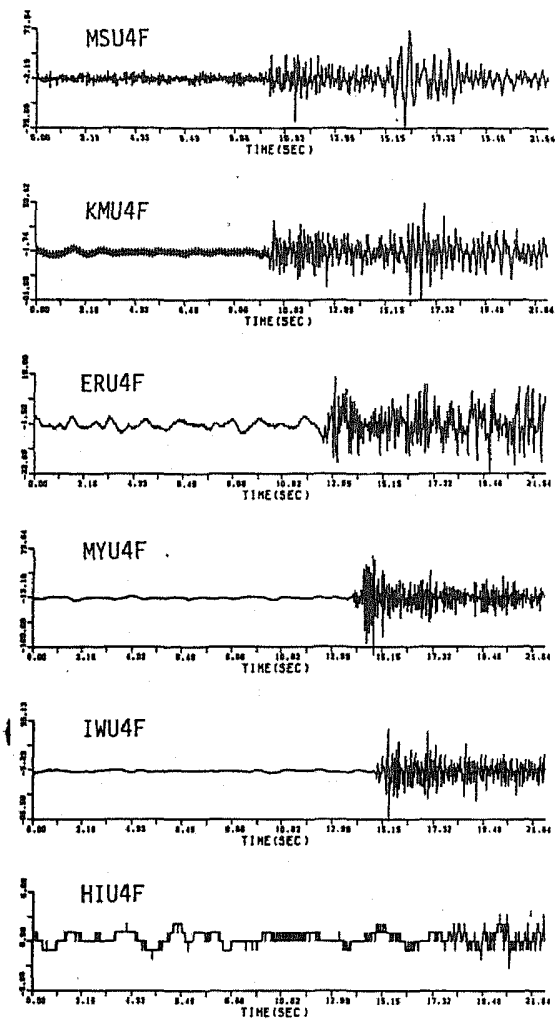

(d)

Fig. 1. (c) Seismograms of foreshock No. 3. (d) Seismograms of foreshock No. 4. Label in upper left-hand corner to seismic records is named after station, component, and number of foreshock.

just looking for the time point that attains the minimum value of the AIC (Akaike Information Criterion) of a locally stationary AR model, namely by finding the model that best fits to the observed data (OZAKI and ToNG, 1975; KITAGAWA and AKAIKE, 1978). The CPU time of this method depends on the number of data (data length) and on the order of the AR model.

The objective of this paper is to develop a computationally efficient algorithm for the fitting of the locally stationary AR model so that it can be applied to the online processing of seismic wave. The procedure is particularly useful for the automatic determination of the arrival time of P- and S-waves. We applied the procedure to some microearthquakes which occurred off Urakawa, Hokkaido, Japan (Fig. 1). At present, this procedure is used in the on-line system of the Research Center for Earthquake Prediction (RCEP) of Hokkaido University (SUZuKi et al., 1986). 


\section{Estimation of Arrival Time by a Locally Stationary AR Model: A Review}

In this section, we will review the use of a time series model for the estimation of arrival time of a seismic wave. The seismometers are under the influence of various kinds of noises such as traffic noise, electronic hum noise, and heavy microtremor. As a result, the observed seismogram shows a random behavior, which is not in general a white noise sequence. This sometimes makes the determination of the arrival time difficult. However, it will be reasonable to assume that the spectral characteristics before and after the arrival of the earthquake are quite different. From the viewpoint of time series modeling, this means that the models for time series before and after the arrival of an earthquake are quite different. Since the spectrum of the time series can be well expressed by an appropriate AR model, it will be also reasonable to use an AR model for each time series. A locally stationary AR model is thus used. In the general definition of a locally stationary AR model (OzAKI and Tong, 1975; KITAGAWA and AKAIKE, 1978), the time interval is divided into $k$ subintervals. However, for the estimation of the arrival time, it seems sufficient to use the following simple model for which the time interval is divided into two parts (YокотA et al., 1981).

We assume that we have a time series $\left\{x_{n} ; n=1, \cdots, N\right\}$. This series can be divided into two subseries and each of them can be expressed by an AR model:

$$
\begin{array}{ll}
x_{n}=\sum_{m=1}^{M(1)} a_{m}^{1} x_{n-m}+\varepsilon_{n}^{1}, & \left(1 \leqq n<p_{2}\right), \\
x_{n}=\sum_{m=1}^{M(2)} a_{m}^{2} x_{n-m}+\varepsilon_{n}^{2}, & \left(p_{2} \leqq n \leqq N\right),
\end{array}
$$

where $\varepsilon_{n}^{i}$ is a Gaussian white noise with mean zero and variance $\sigma_{i}^{2}$; $a_{m}^{i}$ is the autoregressive coefficient, and $M(i)$ is the order of the $i$-th model. It should be noted that $p_{2}$ corresponds to the unknown arrival time. The above two AR models, a background motion model, and an earthquake model constitute our locally stationary AR model for the estimation of P-wave arrival time.

Ignoring the initial distribution, the approximate likelihood of the locally stationary AR model is given by

$$
\prod_{i=1}^{2}\left(\frac{1}{2 \pi \sigma_{i}^{2}}\right)^{\frac{N_{i}}{2}} \exp \left\{-\frac{1}{2 \sigma_{i}^{2}} \sum_{n=p_{i}}^{q_{i}}\left(x_{n}-\sum_{m=1}^{M(i)} a_{m}^{i} x_{n-m}\right)^{2}\right\},
$$

where $q_{0}=M(1), q_{1}=p_{2}-1, q_{2}=N, p_{1}=M(1)+1, N_{i}=q_{i}-q_{i-1}$. Thus by denoting $a^{i}=\left(a_{1}^{i}, \cdots, a_{M(i)}^{i}\right)$, the approximate $\log$ likelihood is given by

$$
\begin{aligned}
& l\left(p_{2}, M(i), a^{i}, \sigma_{i}^{2}(i=1,2)\right) \\
& \quad=-\frac{1}{2} \sum_{i=1}^{2}\left\{N_{i} \log 2 \pi \sigma_{i}^{2}+\frac{1}{\sigma_{i}^{2}} \sum_{n=p_{i}}^{q_{i}}\left(x_{n}-\sum_{m=1}^{M(i)} a_{m}^{i} x_{n-m}\right)^{2}\right\} .
\end{aligned}
$$


For arbitrarily given $a_{m}^{i}$ 's, the maximum of the $\log$ likelihood $l$ is attained at

$$
\sigma_{i}^{2}=\frac{1}{N_{i}} \sum_{n=p_{i}}^{q_{i}}\left(x_{n}-\sum_{m=1}^{M(i)} a_{m}^{i} x_{n-m}\right)^{2} .
$$

Therefore, by substituting Eqs. (4) into (3), the log likelihood function for the estimation of $a_{m}^{i}$ is reduced to the following form:

$$
\begin{aligned}
l^{*}\left(p_{2},\right. & \left.M(i), a_{m}^{i}(i=1,2)\right) \\
& =-\frac{1}{2} \sum_{i=1}^{2}\left\{N_{i} \log 2 \pi \sigma_{i}^{2}+N_{i}\right\} \\
& =-\frac{\left(N-q_{0}\right)}{2}(1+\log 2 \pi)-\frac{1}{2} \sum_{i=1}^{2} N_{i} \log \sigma_{i}^{2} .
\end{aligned}
$$

The maximum likelihood estimate of $a_{m}^{i}$ is obtained by maximizing Eq. (5) or equivalently minimizing $\sigma_{i}^{2}$ with respect to $a_{m}^{i}$ 's. It should be noted that the maximum likelihood estimate of two AR models is obtained by fitting two AR models independently to $\left\{x_{p_{1}}, \cdots, x_{p_{2}-1}\right\}$ and $\left\{x_{p_{2}}, \cdots, x_{N}\right\}$ by the least squares method. A computationally efficient Householder method used for AR model fitting is shown in Appendix 1.

So far we have assumed that the arrival time $p_{1}$ and the model order $M(i)$ are given. But in practice, they are unknown and have to be determined from the data. We will determine these unknown constants so that the corresponding model best fits to the data. This can be realized by minimizing the AIC criterion. AIC (Akaike Information Criterion) was proposed for the selection of the best statistical model (AKAIKE, 1973). Since AIC is obtained by estimating the Kullback-Leibler information number (KULLBACK and LEIBLER, 1951) of the true distribution with respect to the assumed model, it can be considered as a good measure of the badness of the estimated model.

In the case of the locally stationary AR model, AIC is given by

$$
\begin{aligned}
\mathrm{AIC} & =-2(\text { maximum } \log \text { likelihood })+2 \text { (number of parameters) } \\
& =\left(N-q_{0}\right)(1+\log 2 \pi) \sum_{i=1}^{2}\left\{N_{i} \log \hat{\sigma}_{i}^{2}+M(i)+1\right\}
\end{aligned}
$$

In the following argument, it is indispensable to use one and the some data set. We will thus set $q_{0}=\max M(1)$ instead of $M(1)$, and in that case. We can ignore the first term of Eq. (6) since it is common to all possible models.

It should be noted that $\hat{\sigma}_{i}^{2}$ depends on the selection of $p_{2}$ and $M(i)$. For fixed $p_{2}$, AIC is a function of the model orders, $M(1)$ and $M(2)$. Therefore by minimizing AIC, we can determine the best selection of orders. By substituting these into Eq. (6), the AIC becomes a function of only $p_{2}$. We will denote this $\mathrm{AIC}_{p_{2}}$. Then, by finding the $p_{2}$ which attains the minimum of AIC, we can get the best (in the sense of AIC) estimate of the arrival time of the seismic waves. We will call this the minimum 
AIC estimate of the arrival time.

Incidentally, $M(1), a_{m}^{1}, \sigma_{1}^{2}$, and $M(2), a_{m}^{2}, \sigma_{2}^{2}$ which correspond to this arrival time are the minimum AIC estimates of background and seismic wave models, respectively. By substituting these estimates into the theoretical relation between the AR model and the power spectrum,

$$
p_{j}(f)=\frac{\sigma_{j}^{2}}{\left|1-\sum_{m=1}^{M(j)} a_{m} \exp (-2 \pi i m f)\right|^{2}}, \quad(j=1,2)
$$

we can get the power spectra of background noise and seismic wave (KITAGAWA and TAKANAMI, 1985). Here $f$ is frequency.

\section{A Computationally Efficient Implementation of Least Squares Fitting of a Locally Stationary AR Model}

In order to find the best estimate of the arrival time, we have to find the best noise model and the best earthquake model for each possible division of the time interval, namely for all $n_{p_{2}}\left(n_{0} \leqq n_{p_{2}} \leqq n_{1}\right)$. In this section, we will present a computationally efficient procedure based on Householder transformation (Appendix 1) that can yield these models successively.

To be specific, we assume that we have a time series $\left\{x_{1}, \cdots, x_{N}\right\}$ and prior to the analysis we know that the arrival time is on the interval $\left[n_{0}, n_{1}\right]$. It is also assumed that the required resolution is $p$ points, namely we have to fit model for each possible division, $n_{0}, n_{0}+p, \cdots, n_{0}+l p \equiv n_{1}$. We first consider fitting the AR model to the data set $\left\{x_{1}, \cdots, x_{n_{0}}\right\}$. For this, we define the following $\left(n_{0}-k\right) \times(k+1)$ matrix $X_{0}$ :

$$
\boldsymbol{X}_{0}=\left[\begin{array}{lllll}
x_{k} & x_{k-1} & \cdots & x_{1} & x_{k+1} \\
x_{k+1} & x_{k} & \cdots & x_{2} & x_{k+2} \\
\vdots & \vdots & \vdots: \vdots & \vdots \\
x_{n_{0}-1} & x_{n_{0}-2} & \cdots & x_{n_{0}-k} & x_{n_{0}}
\end{array}\right]
$$

The application of the Householder transformation to the matrix $X_{0}$ yields the following upper triangular matrix $\boldsymbol{R}_{0}$ :

$$
\boldsymbol{U} \boldsymbol{X}_{0}=\left[\begin{array}{lllll}
r_{11} & r_{12} & \cdots & r_{1 k} & r_{1, k+1} \\
& r_{22} & \cdots & r_{2 k} & r_{2, k+1} \\
& & \ddots & \vdots & \vdots \\
& & & \vdots & \cdot \\
& & & & r_{k+1, k+1}
\end{array}\right]=\boldsymbol{R}_{0}
$$

Using this upper triangular matrix $\boldsymbol{R}_{0}$, the variance of the noises and AIC's of the AR models with order $j(j=0, \cdots, k)$ are obtained by (see Appendix 1): 


$$
\begin{gathered}
\sigma_{0}^{2}(j)=\frac{1}{n_{0}-k} \sum_{i=j+1}^{k+1} r_{i, k+1}^{2} \quad(j=0,1, \cdots, k), \\
\operatorname{AIC}_{0}(j)=\left(n_{0}-k\right) \log \sigma_{0}^{2}(j)+2(j+1) \quad(j=0,1, \cdots, k) .
\end{gathered}
$$

For fixed $n_{0}, \operatorname{AIC}_{0}(j)$ is the criterion for the selection of best $\mathrm{AR}$ order $M(j)$ for the data set $\left(x_{1}, \cdots, x_{n_{0}}\right\}$. We then define:

$$
\operatorname{AIC}_{0}^{N}=\min _{j}\left\{\operatorname{AIC}_{0}(j)\right\} \quad(j=0, \cdots, k) .
$$

$\mathrm{AIC}_{0}^{N}$ is the AIC of the best noise model obtained under the assumption that the arrival time is $n_{0}+1$ and will be used later for the estimation of arrival time.

Next we will fit an AR model to the augmented data set $\left\{x_{1}, \cdots, x_{n_{0}+p}\right\}$. Obviously this can be done by defining $\left(n_{0}+p-k\right) \times(k+1)$ matrix $\boldsymbol{X}_{1}$ and reducing to an upper triangular form, $\boldsymbol{S}_{1}$, by a Householder transformation as follows:

$$
\begin{aligned}
& X_{1}=\left[\begin{array}{lll}
x_{k} & \cdots x_{1} & x_{k+1} \\
x_{k+1} & \cdots x_{2} & x_{k+2} \\
\vdots & \vdots: \vdots & \vdots \\
x_{n_{0}+p-1} & \cdots x_{n_{0}+p-k} & x_{n_{0}+p}
\end{array}\right] \\
& \boldsymbol{U}_{1} \boldsymbol{X}_{1}=\left[\begin{array}{llll}
s_{11} & s_{12} & \cdots & s_{1, k+1} \\
& s_{22} & \cdots & s_{2, k+1} \\
& 0 & \ddots & \vdots \\
& & & s_{k+1, k+1}
\end{array}\right]=S_{1}
\end{aligned}
$$

However, by the property of the orthogonal transformation, it can be seen that one and the same upper triangular matrix is obtained by first organizing a $(k+1+p) \times$ $(k+1)$ matrix $\boldsymbol{R}_{1}$ and then reducing to an upper triangular form $\boldsymbol{S}_{1}{ }^{\prime}$ by a proper Householder transformation as follows:

$$
\begin{gathered}
\boldsymbol{R}_{1}=\left[\begin{array}{llll}
r_{11} & \cdots & r_{1 k} & r_{1, k+1} \\
& & \vdots & \\
& & r_{k k} & r_{k, k+1} \\
& 0 & & r_{k+1, k+1} \\
x_{n_{0}} & \cdots & x_{n_{0}+k-1} & x_{n_{0}+1} \\
\vdots & & \vdots & \vdots \\
x_{n_{0}+p-1} & \cdots & x_{n_{0}+p-k} & x_{n_{0}+p}
\end{array}\right], \\
\boldsymbol{U}_{1}{ }^{\prime} \boldsymbol{R}_{1}=\left[\begin{array}{llll}
s_{11} & \cdots & s_{1, k+1} \\
& \cdots & \vdots \\
& 0 & s_{k+1, k+1}
\end{array}\right]=S_{1}{ }^{\prime} .
\end{gathered}
$$

This is a very important modification. Note that the number of rows of the matrix 
$X_{1}$ and $S_{1}$ are $n_{0}+p-k$ whereas those of $\boldsymbol{R}_{1}$ and $S_{1}{ }^{\prime}$ are $k+p+1$. The variance of the noise and AIC of the AR models for the data set $\left\{x_{1}, \cdots, x_{n_{0}+p}\right\}$ is obtained by

$$
\begin{gathered}
\sigma_{1}^{2}(j)=\frac{1}{n_{0}-k+p} \sum_{i=j+1}^{k+1} s_{i, k+1}^{2} \\
\operatorname{AIC}_{1}(j)=\left(n_{0}-k+p\right) \log \sigma_{n_{0}+p}^{2}(j)+2(j+1) \\
\operatorname{AIC}_{1}^{N}=\min _{j} \operatorname{AIC}_{1}(j) .
\end{gathered}
$$

We can repeat this method until we get entire AR models and corresponding AIC's for the data set $\left\{x_{1}, \cdots, x_{n_{0}}\right\},\left\{x_{1}, \cdots, x_{n_{0}+p}\right\}, \cdots,\left\{x_{1}, \cdots, x_{n_{1}}\right\}$. As the result, we obtained $\mathrm{AIC}_{0}^{N}, \mathrm{AIC}_{1}^{N}, \cdots, \mathrm{AIC}_{l}^{N}$.

In the same way, we can also get entire earthquake models. Namely, we first fit an AR model to the data set $\left\{x_{n_{1}-p+1}, \cdots, x_{N}\right\}$, and obtain $\mathrm{AIC}_{l}^{P} . \mathrm{AIC}_{l-1}^{P}$ is then obtained by augmenting $\left\{x_{n_{1}-2 p+1}, \cdots, x_{n_{1}-p}\right\}$ in the same way as Eq. (13). Repeating this method $l$ times, we obtain $\mathrm{AIC}_{0}^{P}, \cdots, \mathrm{AIC}_{l}^{P}$. Then the sum of two AIC's is as follows:

$$
\mathrm{AIC}_{i}=\mathrm{AIC}_{i}^{N}+\mathrm{AIC}_{i}^{P},
$$

which expresses the AIC of the locally stationary AR model for which the time interval is assumed to be divided into $\left[1, n_{0}+i p\right]$ and $\left[n_{0}+i p+1, N\right]$. This means that the arrival time is $n_{0}+i p+1$. Thus the best estimate of arrival time can be found by selecting the minimum among $\mathrm{AIC}_{0}, \cdots, \mathrm{AIC}_{l}$.

For the Householder transformation of a $n \times(k+1)$ matrix, the amount of necessary multiplication (and the addition) can be evaluated as

$$
\begin{aligned}
\sum_{i=1}^{k+1}(n+1-i)(k+2-i)=\sum_{i=1}^{k+1}\left\{i^{2}-(n+k+3) i+(n+1)(k+2)\right\} \\
= \\
=\frac{(k+1)(k+2)(2 k+3)}{6}-(n+k+3) \frac{(k+1)(k+2)}{2}+(n+1)(k+2)(k+1) \\
\approx \\
\approx \frac{1}{2} n k^{2}
\end{aligned}
$$

where $n$ is the number of rows of the matrix $X_{1}$ and depends on the assumed arrival time. Therefore the total amount of multiplication (and addition) for the comparison of all possible locally stationary AR models by the ordinary method (12) is roughly of the order of 


$$
\begin{aligned}
\sum_{i=0}^{l} & \frac{1}{2}\left(n_{0}+i p\right) k^{2}+\sum_{i=0}^{l} \frac{1}{2}\left(N-n_{0}-i p\right) k^{2} \\
& =\frac{1}{2}\left\{n_{0} k^{2}(l+1)+\frac{1}{2} p k^{2} l(l+1)\right\}+\frac{1}{2}\left\{\left(N-n_{0}\right) k^{2}(l+1)-\frac{1}{2} p k^{2} l(l+1)\right\} \\
& =\frac{1}{2} N k^{2}(l+1) \\
& \approx \frac{N k^{2}\left(n_{1}-n_{0}\right)}{2 p}
\end{aligned}
$$

On the other hand, for the Householder transformation Eq. (13), the amount of multiplication is

$$
\begin{aligned}
& \sum_{i=1}^{k+1}(p+1)(k+2-i)=\sum_{i=1}^{k+1}\{(p+1)(k+2)-(p+1) i\} \\
& =(p+1)(k+2)(k+1)-\frac{1}{2}(p+1)(k+1)(k+2) \\
& \quad=\frac{1}{2}(p+1)(k+1)(k+2) \\
& \quad \approx \frac{1}{2} p k^{2} .
\end{aligned}
$$

Therefore the total amount of multiplication by the present method is

$$
\begin{aligned}
\frac{1}{2} n_{0} & k^{2}+\sum_{i=1}^{l} \frac{1}{2} i p k^{2}+\frac{1}{2}\left(N-n_{1}\right) k^{2}+\sum_{i=1}^{l} \frac{1}{2} i p k^{2} \\
= & \frac{1}{2}\left(N+n_{0}-n_{1}\right) k^{2}+p k^{2} l \\
= & \frac{1}{2}\left(N+n_{0}-n_{1}\right) k^{2}+k^{2}\left(n_{1}-n_{0}\right) \\
= & \frac{1}{2} N k^{2}+\frac{1}{2} k^{2}\left(n_{1}-n_{0}\right) \quad\left(<N k^{2}\right) .
\end{aligned}
$$

It should be noted that the amount of operations for the proposed method does not depend on the number of models, $l$. For example, if $N=3,000, n_{0}=1,000, n_{1}=2,000$, $k=10$, and $p=2$, the necessary amount of multiplications are respectively

$$
(3,000)\left(10^{2}\right)(1,000) / 4=7.5 \times 10^{7},
$$

and

$$
\frac{1}{2}(3,000)\left(10^{2}\right)+\frac{1}{2}\left(10^{2}\right)(1,000)=2 \times 10^{5} .
$$




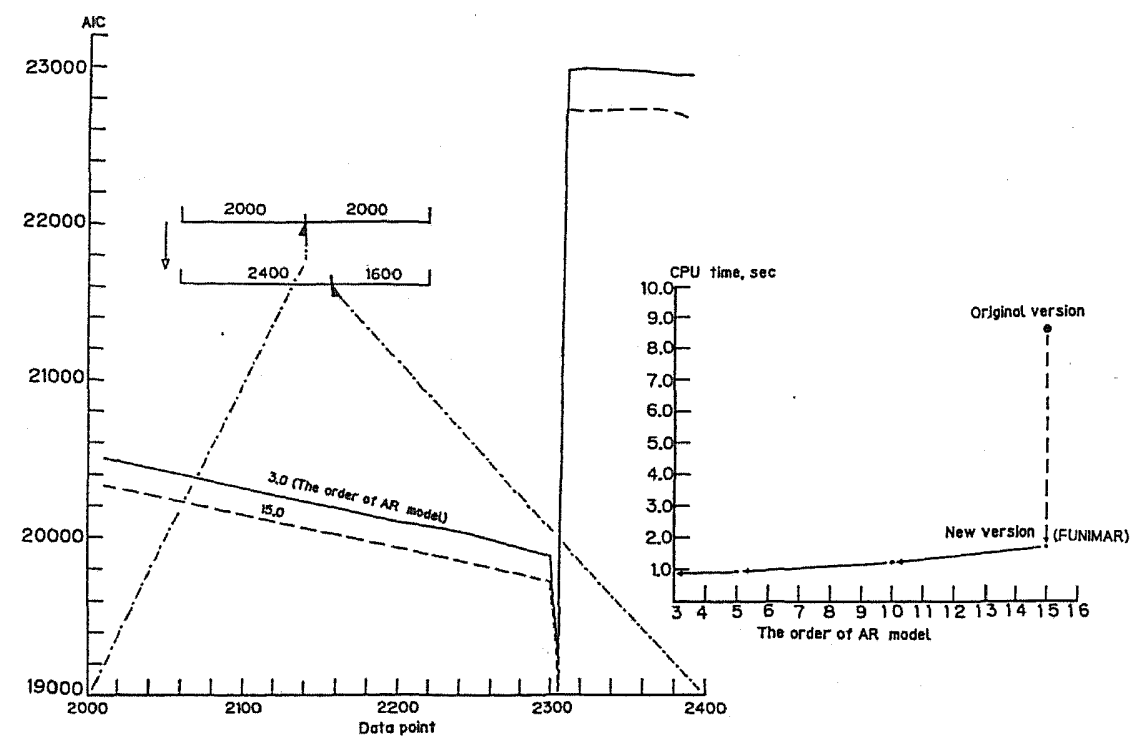

Fig. 2. Comparison of CPU times by unmodified procedure and by FUNIMAR. Right: Comparison of the CPU times by traditional least squares computation by Eq. (12), and by the newly modified least squares computation by Eq. (13), FUNIMAR. Further, comparison of the CPU times according to the order of AR model is shown by using the procedure FUNIMAR. Left: Examples of AIC curves of the AR model of order 3 and of the AR model of order 15 (dashed line). Test data is an up-down component seismogram recorded at station ESH.

Therefore, by the present procedure, the amount of computation for Householder transformation is reduced to about $1 / 400$ th of the original procedure. The Fortran program FUNIMAR is developed to implement this procedure. The actual CPU time for FUNIMAR is approximately one tenth of that required by the unmodified procedure, as shown in Fig. 2.

\section{Application of the Procedure to the Real Seismograms}

The procedure developed in the previous section has been applied to the seismograms observed at the stations of RCEP. In the recording system, ground velocity signal from the geophone at each station is first digitized by an 8 bit nonlinear AD converter at the rate of 92.3 samples/s $(2,400 \mathrm{bps} / 26 \mathrm{bit})$; waves with frequency above $30 \mathrm{~Hz}$ were eliminated by an anti-aliasing filter (Butterworth filter of order 6), and transformed to pulse code modulation (PCM) data. These PCM data were transmitted to the central recording station of Hokkaido University (e.g., MAEDA, 1978) and recorded on a magnetic tape by a high-density data recorder (HDDR of Yamatake-Honeywell Co.) which drives continuously for two days. 
Table 1. Source parameters of the events used in the present study.

\begin{tabular}{|c|c|c|c|c|c|c|c|c|}
\hline Date & (h) & $\begin{array}{l}\text { Time } \\
\text { (min) }\end{array}$ & (s) & $\begin{array}{l}\text { Long. } \\
\text { (E) }\end{array}$ & $\begin{array}{l}\text { Lat. } \\
\left({ }^{\circ} \mathrm{E}\right)\end{array}$ & $\begin{array}{c}\text { Depth } \\
(\mathrm{km})\end{array}$ & $M$ & Event No. \\
\hline Mar. 21, 1982 & 07 & 45 & 53.0 & 142.557 & 42.158 & 31.0 & 1.9 & Foreshock No. 1 \\
\hline Mar. 21, 1982 & 08 & 42 & 52.0 & 142.555 & 42.131 & 26.0 & 2.0 & Foreshock No. 2 \\
\hline Mar. 21, 1982 & 08 & 49 & 20.7 & 142.561 & 42.158 & 33.7 & 2.1 & Foreshock No. 3 \\
\hline Mar. 21, 1982 & 09 & 33 & 15.0 & 142.574 & 42.133 & 31.1 & 2.3 & Foreshock No. 4 \\
\hline Dec. 12,1982 & 12 & 04 & 24.0 & 142.380 & 42.090 & 18.8 & 2.6 & Aftershock No. 1 \\
\hline
\end{tabular}

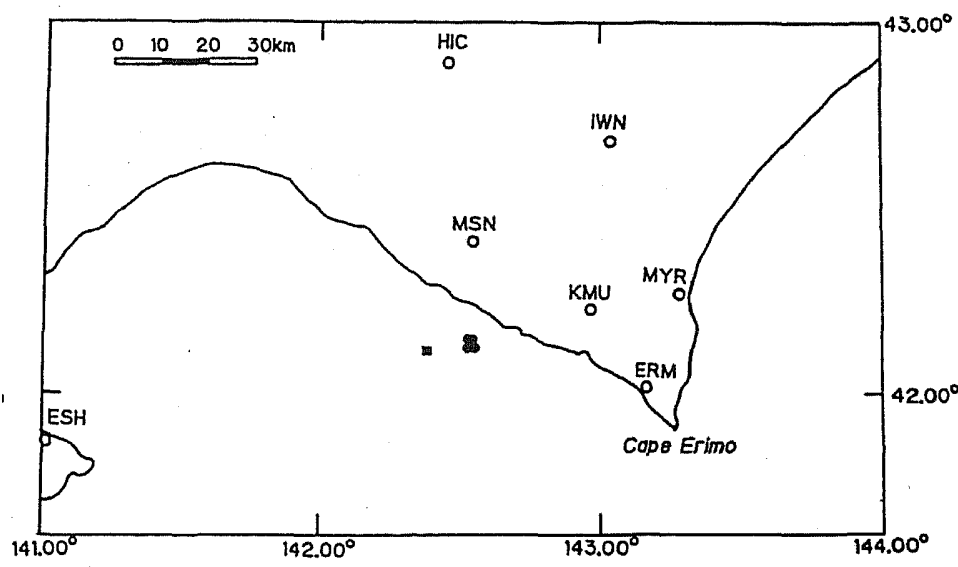

Fig. 3. Locations of recording stations (open circles), foreshock epicenters (solid circles), and aftershock epicenter (solid square) of the 1982 Urakawa-Oki Earthquake used in the present study.

For the illustration of the new procedure, we used seismograms of four foreshocks of the 1982 off-Urakawa Earthquake of M 7.1 (SAKAI, 1983) recorded at six stations in the Hidaka region and that of an aftershock recorded at the station near Esan. In particular, much attention was paid to noisy seismograms, which are contaminated with several kinds of noises, such as sudden traffic noise, electronic hum, or relatively stronger microseisms (see Fig. 1). Table 1 summarizes the information about the earthquakes used in the present study. The magnitudes of these earthquakes were about 2.0. Locations of the epicenters and the seismic stations are shown in Fig. 3. They are located very close to the main shock.

We also checked the feasibility of estimating the arrival time of S-wave. Usually the first portion of the S-wave train is superimposed on the strong coda wave induced by the $\mathrm{P}$-wave. Thus it is not easy to visually determine the onset time of the S-wave. The seismogram recorded at Esan (ESH) is used to examine whether the current procedure can pick out the correct onset time of the S-wave disturbed by such P-coda waves. 
$(F=0.05 \cdot 0.08 \cdot 7 \cdot 0 \cdot 10.0 \mathrm{HZ})$
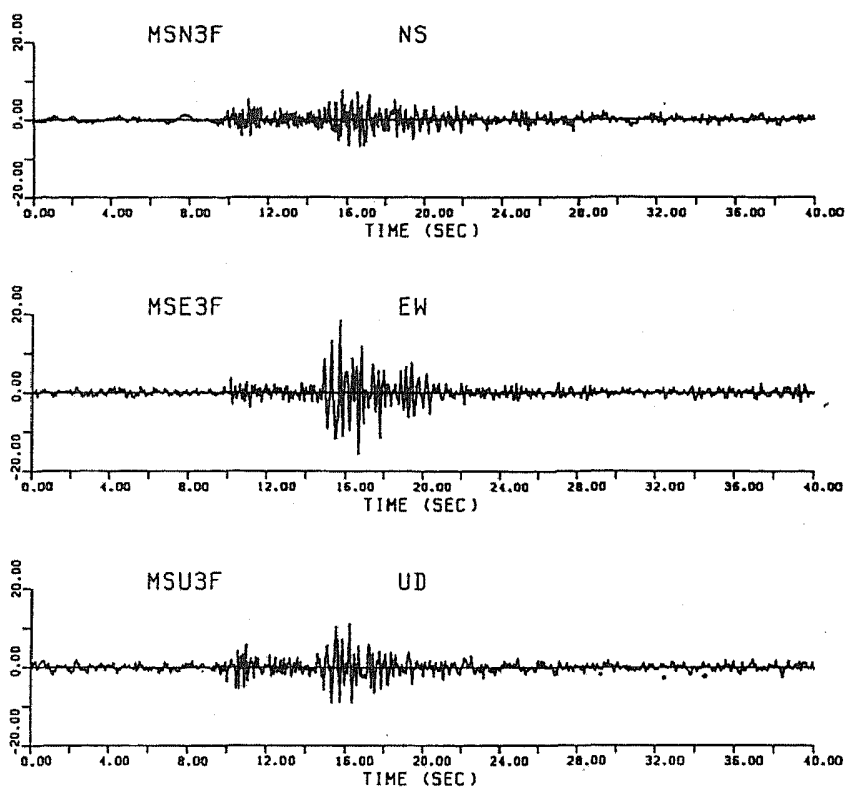

Fig. 4. Filtered three-component seismograms of foreshock No. 3 at station MSN. The frequency of the band-pass filter ranges between 0.08 and $7.0 \mathrm{~Hz}$. Outside of their frequencies, amplitudes of spectra are tapered by half-cosine function. Note its unfiltered up-down component seismogram shown in the top of Fig. 1(c).

\subsection{The wave buried in traffic noise recorded at Misono station}

The distances from the epicenters to the nearest station, Misono (MSN), are about $30 \mathrm{~km}$. The station MSN is located near a road and is occasionally subject to traffic noises (See MSU3F Event 3 of Fig. 1). We first applied the band-pass filter of $0.08-7.0 \mathrm{~Hz}$ to enhance seismic signals (Fig. 4). Although the traffic noises are suppressed considerably by this filter, the onset times of P-waves in any of the three components are still ambiguous. We applied the present procedure to the unfiltered data recorded by the up-down component seismograph. $\mathrm{AIC}_{n}$ and the seismogram are shown in Fig. 5. $\mathrm{AIC}_{n}$ indicates the badness of the fit of the locally stationary AR model which assumes that the $\mathrm{P}$-waves arrive at $n_{0}+n p$-th time point. The arrow on this record indicates the arrival time determined by the AIC criterion. In this case the AIC has a clear minimum. It indicates that we can get a good estimate of P-wave onset time. An implication of this result is that at least in this case, the AR model of the seismic signal is considerably different from that of the traffic noise. It should be noted that the procedure can determine the onset time of seismic arrival without prior noise reduction filter. 

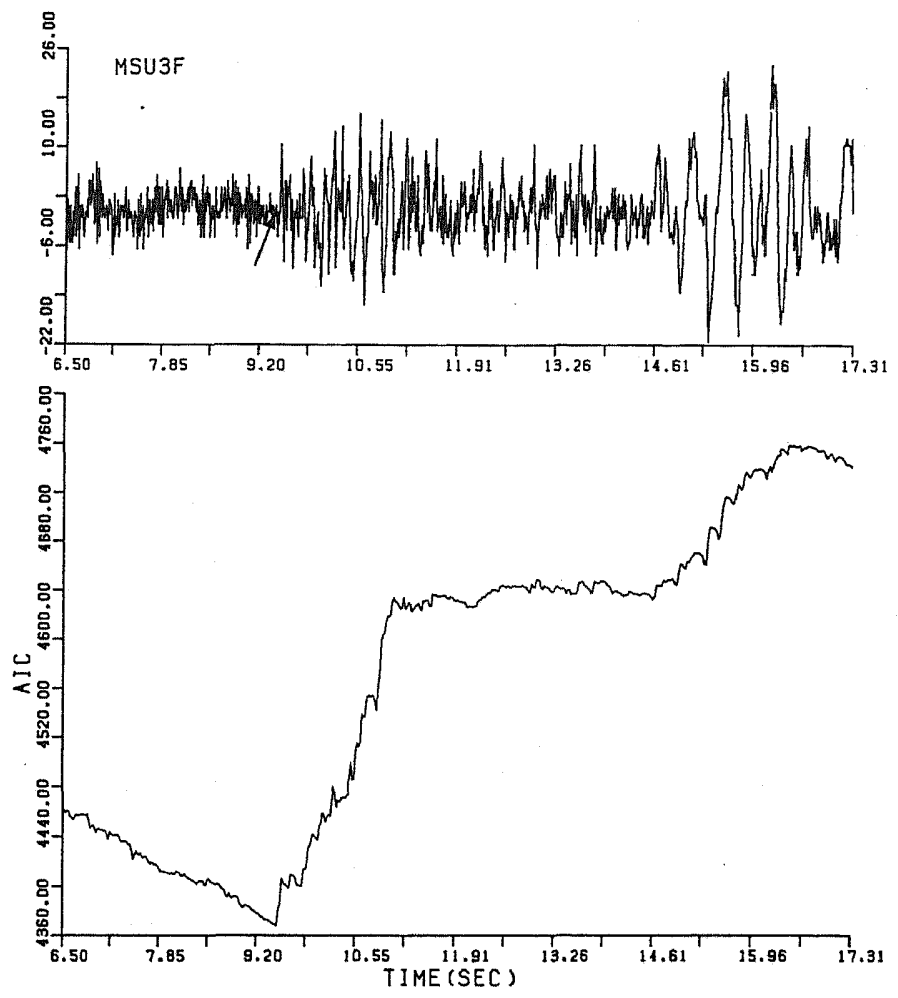

Fig. 5. The determined onset time of P-arrival of the event No. 3 recorded at MSN and AIC obtained from FUNIMAR. An arrow indicates the onset time inferred from minimum AIC.

\subsection{The seismogram superimposed on the strong microtremor noises record- ed at Erimo station}

The station Erimo (ERM) is located at a distance of $0.5 \mathrm{~km}$ from the shoreline of the Pacific and has epicentral distance of about $50 \mathrm{~km}$ from the locations of the foreshocks. The ratios of seismic signal to the background noise are about 0.5 or less. Besides the geometrical spreading, they are strongly affected by attenuation in the crustal structure along the ray paths (e.g., TAKANAMI, 1982). In the ordinary routine work, it is very hard to identify the onset times of such weak P-waves recorded on the paper chart. The AIC values together with the original up-down component seismogram of the event No. 3 are shown in Fig. 6. The arrow indicates the estimated onset time of P-wave, i.e., the time that corresponds to the minimum of AIC. The trace of time versus AIC shows a sharp wedge in the neighborhood of the minimum of AIC. This indicates that if the noise is the microtremor type we may get a good estimate of onset time of $\mathrm{P}$-wave even though the signal-to-noise ratio is as low as 0.5 or less. 


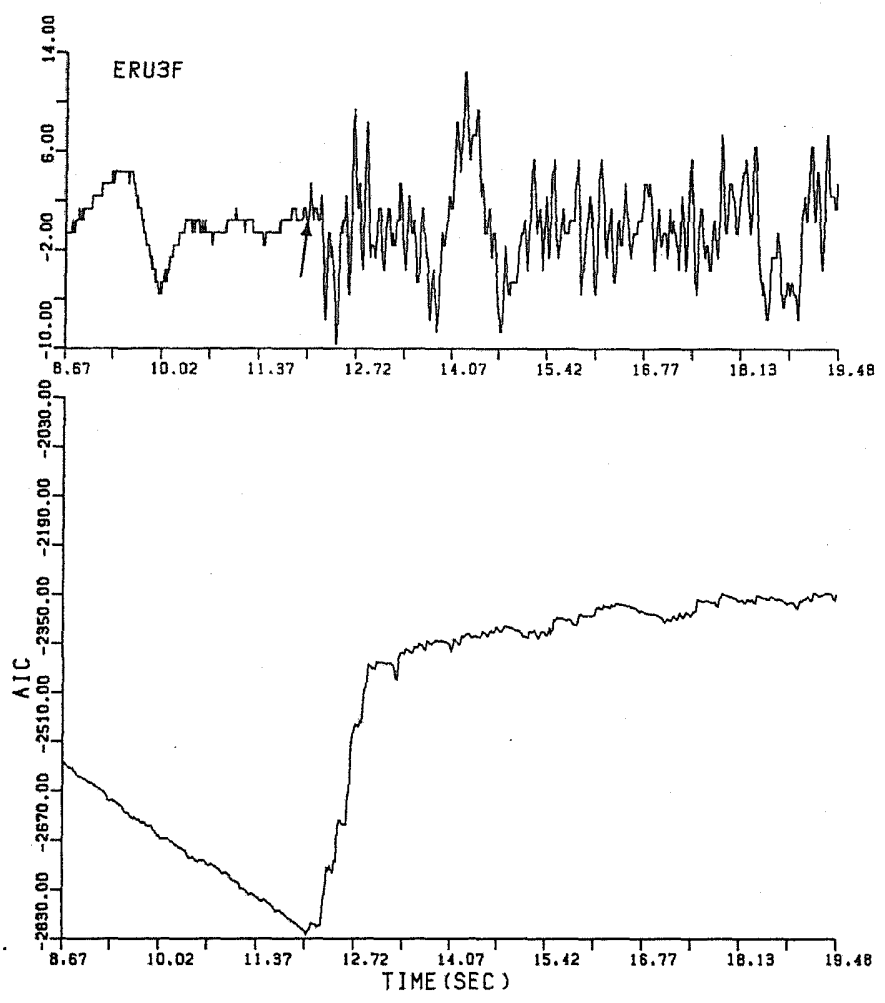

Fig. 6. The determined onset time of P-arrival of the event No. 3 recorded at ERM. Other explanation is the same as in Fig. 5 legend.

\subsection{The weak seismograms recorded at Hidaka station}

The station Hidaka (HIC) is located at a distance of $85 \mathrm{~km}$ from the epicenters of the foreshocks and is located far-off from towns and roads. The amplitudes of the seismograms recorded at station HIC are very weak and almost equal to one LSB (least significant bit) of the digital data and are comparable to those of the background noises due to traffic, machinery, or oceanic sources. The typical seismograms and the corresponding AIC are shown in Fig. 7.

The behavior of AIC is not so monotonous as that of previous examples. Two local minima of AIC are found at 18.18 and $19.40 \mathrm{~s}$ as candidates of the P-arrival time; the arrow on the seismogram corresponds to the smallest one.

4.4 The seismograms with strong hum noise recorded at Kamikineusu station

The station Kamikineusu (KMU) is located at a distance of about $25 \mathrm{~km}$ from the epicenters of the foreshocks. On this occasion, the seismograms obtained at station KMU were contaminated by a strong electronic noise, a hum with a frequency of $50 \mathrm{~Hz}$, which remains even after the application of the Butterworth low 


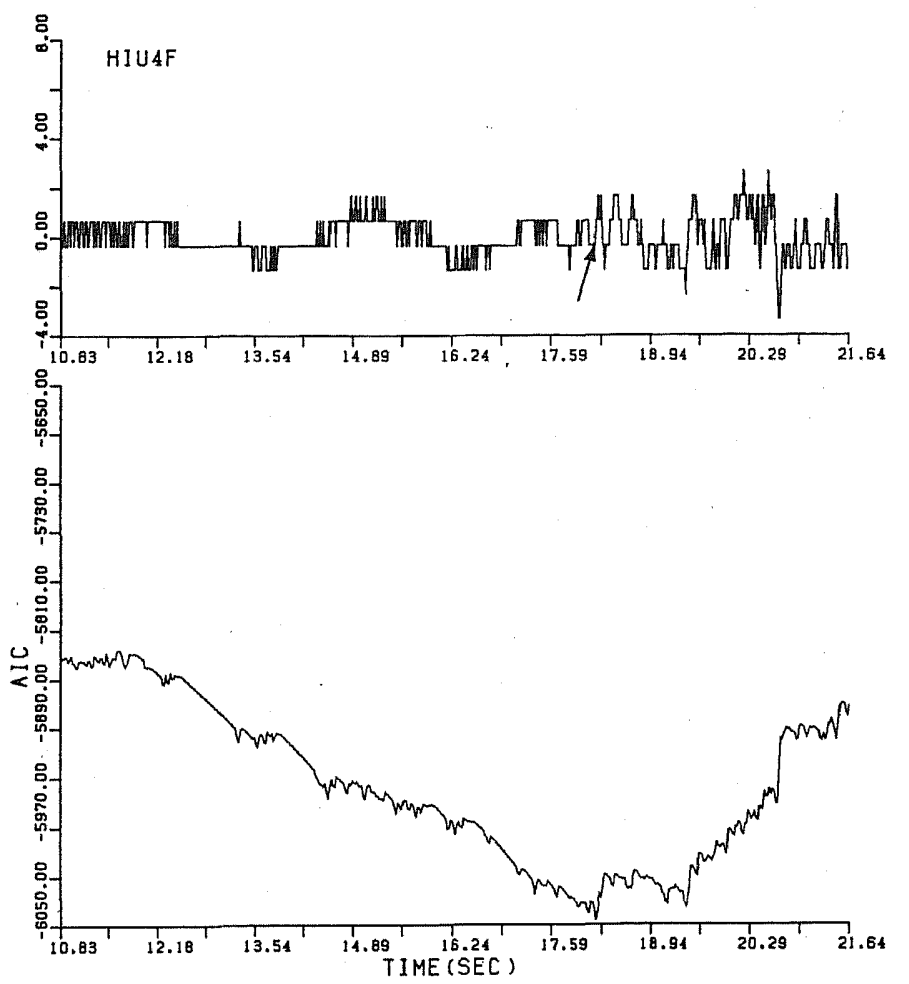

Fig. 7. The determined onset time of P-arrival of the event No. 4 recorded at HIC. Other explanation is the same as in Fig. 5 legend.

pass filters of order 6. As shown in Fig. 8 the seismic signals of the small foreshocks are unclear due to the presence of hum, and it is hard to determine the onset times of $\mathrm{P}$-waves. The arrow in the figure indicates the estimate of the arrival time determined by the minimum AIC procedure. Considering the information from other stations, it was confirmed that the position of the arrow actually indicates the onset time of the first P-arrival. This example is a corroboration that the present procedure works even when strong hum noise is present.

\subsection{Availability for the estimation of the onset time of $S$-wave}

The S-waves of local earthquakes, whose S-P times are not so large, are contaminated by the P-wave trains. However, it is expected that $\mathrm{P}$ - and $\mathrm{S}$-waves have different spectra and thus the locally stationary AR model can distinguish these two waves. Figure 9 represents the seismogram recorded at the station ESH. This station is located west-south-west of the epicenter. The event used here is one of the aftershocks of the off-Urakawa earthquake and occurred on December 12, 1982. Figure 9 represents the two local minima of the AIC. The first local minimum of the AIC corresponds to the onset time of P-wave determined by the AR model of 

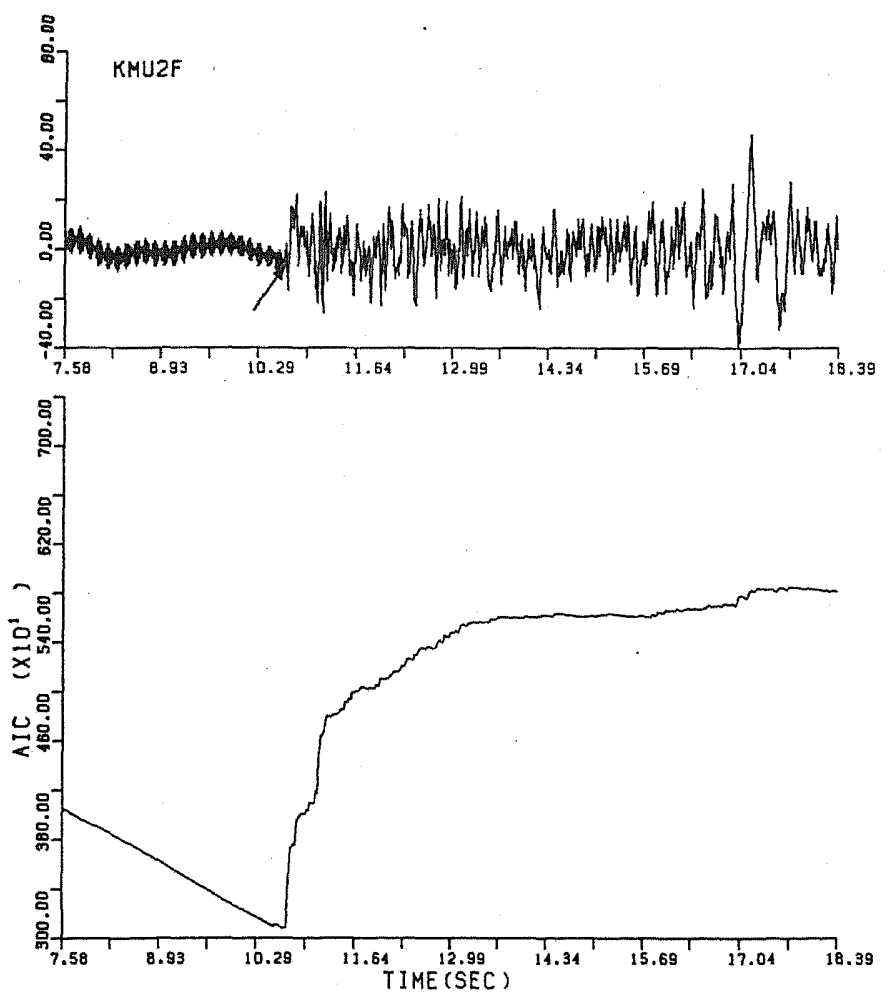

Fig. 8. The determined onset time of P-arrival of the event No. 2 recorded at KMU. Other explanation is the same as in Fig. 5 legend.

$\mathrm{P}$-wave. The second one exactly corresponds to the onset time of S-wave determined by the AR model of S-wave. It is therefore suggested that the procedure FUNIMAR, which has been focused mainly on the decision of the onset time of first P-arrival, can be also useful for the determination of the onset time of the Sarrival.

\section{Discussion and Conclusion}

In the present study, we show a new, efficient, and numerically stable method for the fitting of a locally stationary AR model which is suited to on-line processing. In our method, we used a Householder transformation for the least squares computation. The proposed method was applied to the typical up-down component records of microearthquakes. It was shown that the proposed procedure can reasonably identify the arrival time of seismic wave even when it was contaminated by various kinds of noises such as traffic noise, hum, and when the signal-to-noise ratio is very small (Fig. 10). If the amplitude of seismic signal is greater than the 


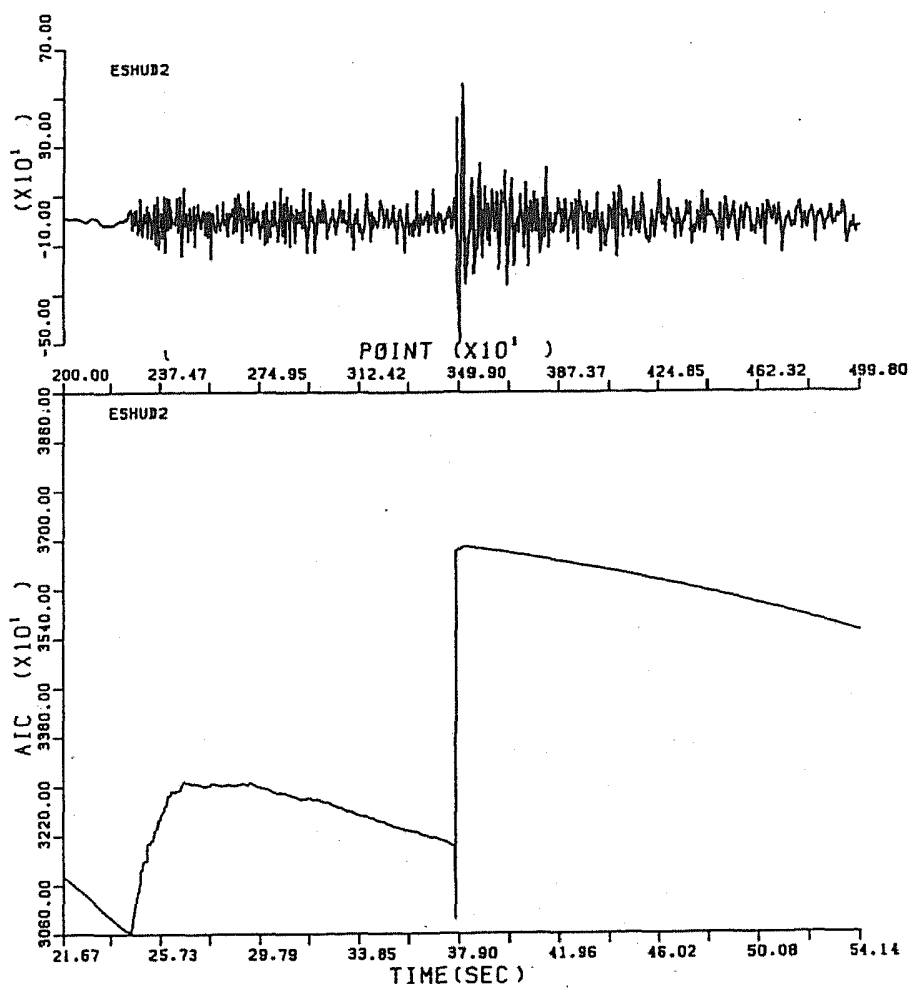

Fig. 9. The seismic record No. 1 of ESH and AIC curve. The two clear minima in the AIC curve indicate the onset times of P-wave and S-wave, respectively.

level of LSB, the present procedure can reasonably determine the onset time of the $\mathrm{P}$-arrival. Even for the case when we have difficulty in determining the onset time of seismic arrival, it could possibly be alleviated by the use of a multi-channel version of the present procedure. Further, by the proper modification of orthogonal transformation, CPU time is significantly reduced. Namely, $O\left(N k^{2} l\right)$ operations are reduced to only $O\left(N k^{2}\right)$, where $N, l$, and $k$ are the length of the data set, the number of models checked, and the upper limit of the order of the AR model, respectively.

By the implementation of the modified procedure for the AR model fitting, it is suggested that the computing times are reduced to about one tenth of the time required by the unmodified one and the AR model with order 5 will be sufficient to determine the onset time of the seismic phase from the background noise without decreasing accuracies. In addition, the onset times of S-waves, which are mixed with the scattered coda waves induced by P-wave, can also be successfully determined by the current method. Morita and Hamaguchi (1984) discussed a mathematical aspect for the confidence interval of the onset time estimate. They concluded that the $90 \%$ confidence interval times were estimated to be 0.2 and 0.8 s for $\mathrm{P}$ and $\mathrm{S}$ onsets 
(a)
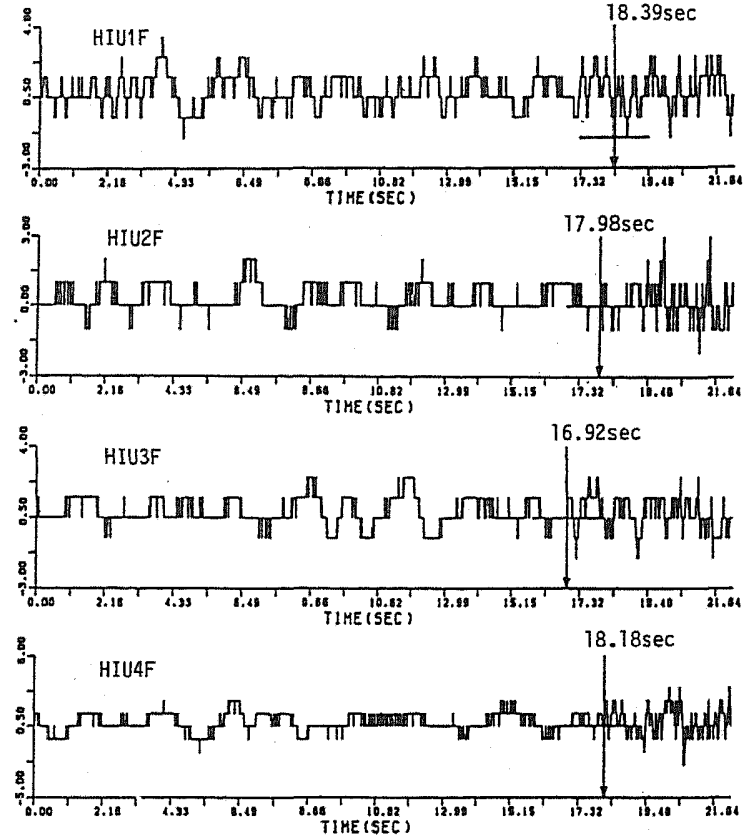

(b)
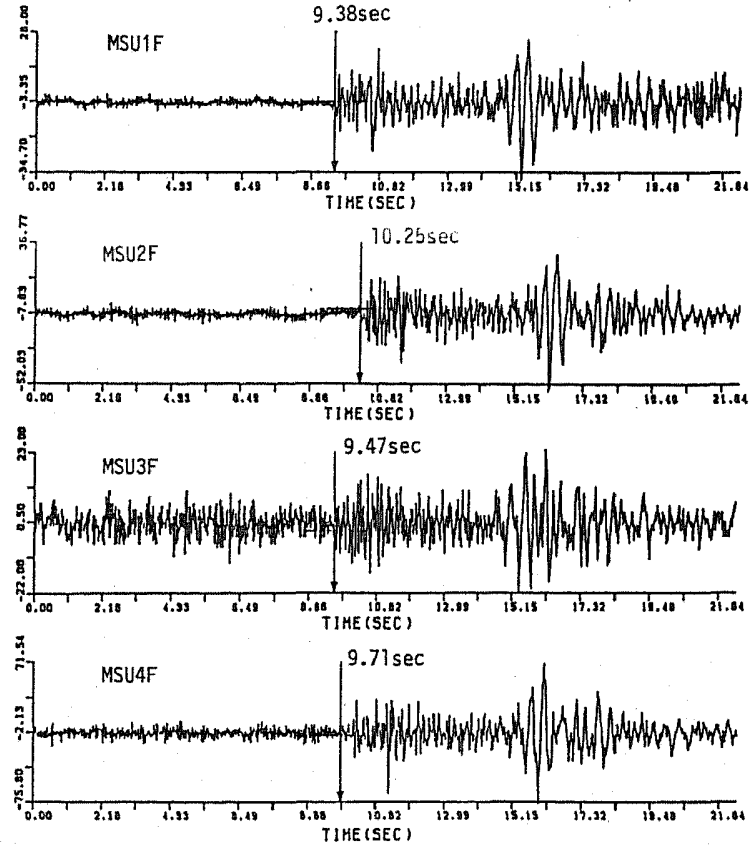

Fig. 10 
(c)
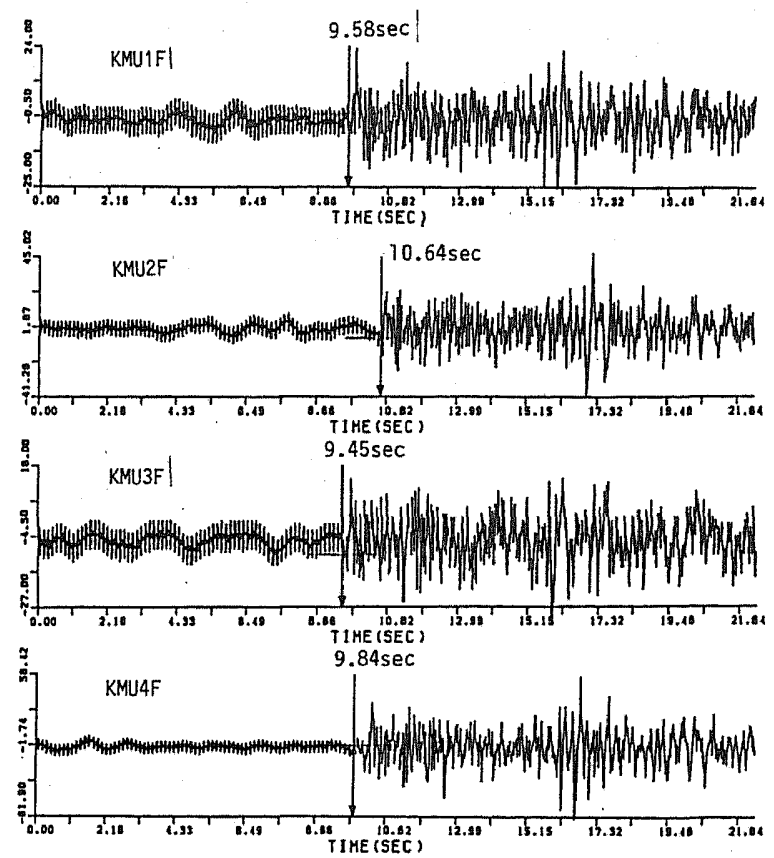

(d)
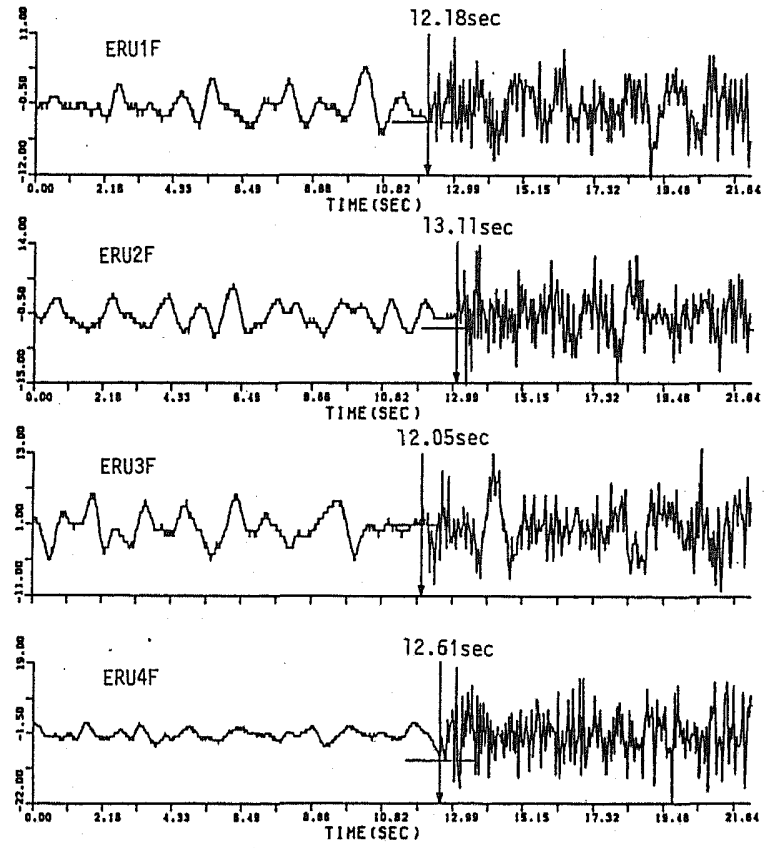

Fig. 10 
(e)
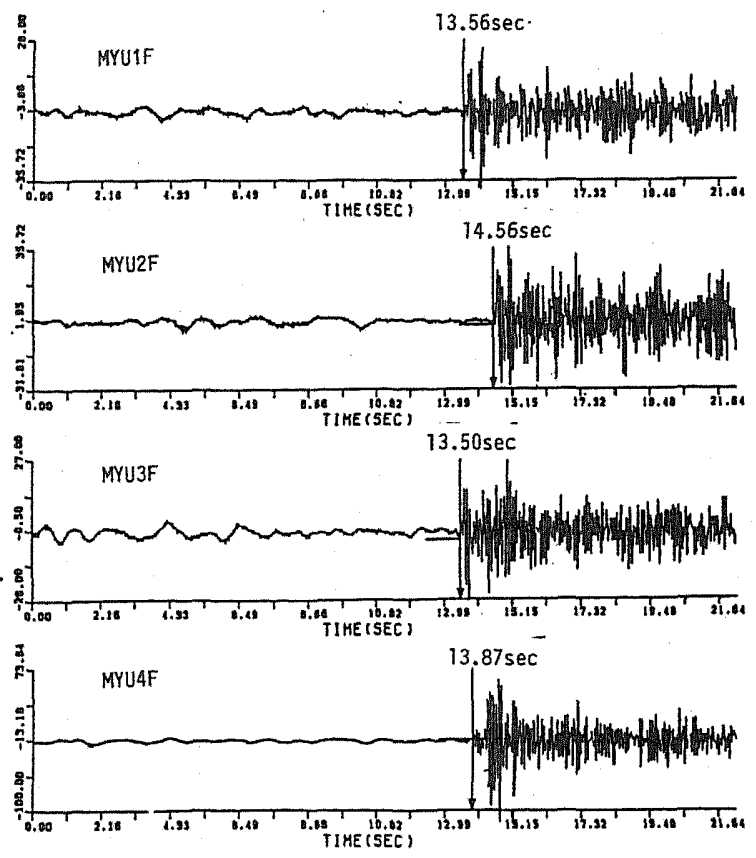

(f)
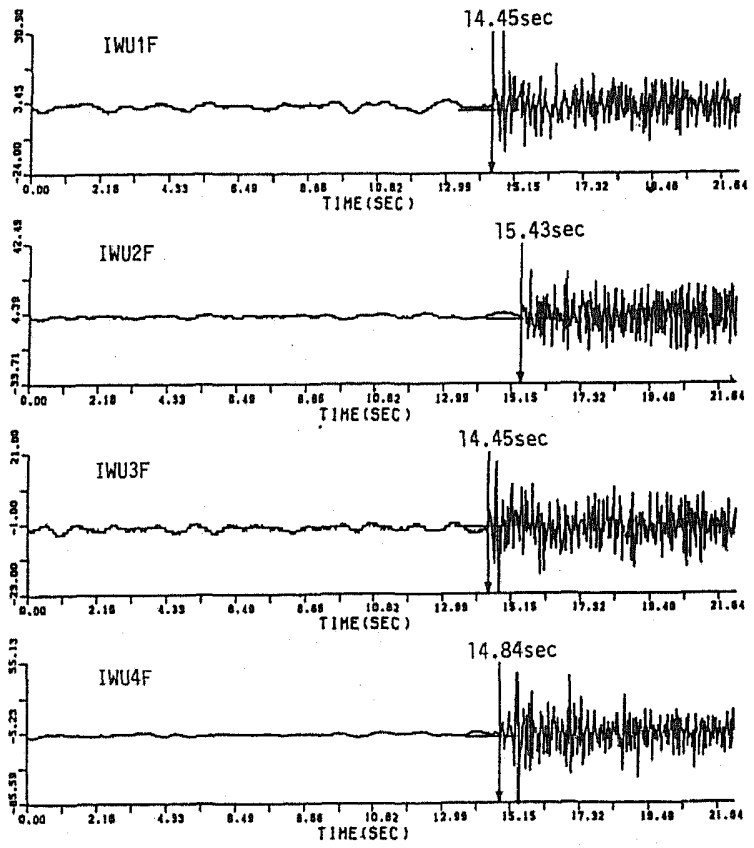

Fig. 10. The onset times determined by using AIC. The determined onset times of P-waves are marked with arrows on seismograms. (a) Station HIC; (b) station MSN; (c) station KMU; (d) station ERM; (e) station MYR; (f) station IWN. 
when the signal-to-noise ratios were about $10 \mathrm{~dB}$. Judging from the present study, the confidence interval of the onset time depends not only on such ratio but also the difference between AR models, i.e., the spectral content of seismic signal and that of background noise.

We are grateful to Prof. H. Akaike of the Institute of Statistical Mathematics for valuable advice. We also thank Prof. H. Okada and the colleagues of the Research Center for Earthquake Prediction for the discussion on the validity of our procedure to the real-time data. Careful and critical reading by the referee is gratefully acknowledged.

\section{REFERENCES}

AKAIKE, H., Information theory and an extension of the maximum likelihood principle, in 2nd International Symposium on Information Theory, ed. B. N. Petrov and F. Csaki, pp. 267-281, Budapest Akademiai Kiado, 1973.

GoluB, G. H., Numerical methods for solving linear least squares problems, Numer. Math., 7, 206-219, 1965.

HAMAGUCH, H. and Y. MORITA, Second-order autoregressive representation of short-period seismic waves, Zisin, Ser. 2, 33, 131-140, 1980 (in Japanese with English abstract).

HAMAGUCHI, $\mathrm{H}$. and $\mathrm{Z}$. SUZUKI, Automatic detection of onset time of microearthquake and its confidence, Report of Grant-in-aid for Scientific Research Project on Natural Disaster (No. 54-2), Ministry of Education, Science and Culture, 62-83, 1979.

Hasegawa, A., N. Umino, A. Yamamoto, and A. Takagi, Automatic event detection and location system of microearthquake observation network, Zisin, Ser. 2, 39, 381-395, 1986 (in Japanese with English abstract).

Kitagawa, G. and H. AkaIke, Procedure for the modeling of non-stationary time series, Ann. Inst. Statist. Math., 30, 351-363, 1978.

Kitagawa, G. and T. TAKanami, Extraction of signal by a time series model and screening out microearthquakes, Signal Processing, 8, 303-314, 1985.

Kullback, S. and R. A. Leibler, On information and sufficiency, Ann. Math. Statist., 22, 79-86, 1951.

MAEDA, I., Telemetry system for observation of microearthquake and crustal movement, Note of the Research Center for Earthquake Prediction, Faculty of Science, Hokkaido University, Vol. 3, 1-7, 1978 (in Japanese).

MAEDA, N., A method for reading and checking phase time in auto-processing system of seismic wave data, Zisin, Ser. 2, 38, 365-379, 1985 (in Japanese with English abstract).

MAEDA, N., On reliability of the results obtained by the method for automatic determination of phase times from seismic wave data ${ }_{2}$ Zisin, Ser. 2, 39, 555-566, 1986 (in Japanese with English abstract).

Morita, Y. and H. Hamaguchi, Automatic detection of S-onset times using twodimensional autoregressive model fitting, Zisin, Ser. 2, 34, 223-240, 1981 (in Japanese with English abstract).

Morita, Y. and H. HamaGuchi, Automatic detection of onset times of seismic waves and its confidence interval using the autoregressive model fitting, Zisin, Ser. 2, 37, 281-293, 1984 (in Japanese with English abstract).

OZAKI, T. and H. TONG, On the fitting of non-stationary autoregressive models in the time 
series analysis, Proceeding of the 8th Hawaii International Conference on System Science, Western Periodical Company, 1975.

SAKAI, Y., General report on the Urakawa-Oki Japan, Earthquake, of March 21, 1982, Report of Grant-in-aid for Scientific Research Project on Natural Disaster (No. 56020014 and No. 57020018), Ministry of Education, Science and Culture, 1983.

SHIRAI, K. and I. ToKuHIRo, Detection of seismic onsets, Zisin, Ser. 2, 32, 141-148, 1979 (in Japanese with English abstract).

Suzuki, S., T. Takanami, Y. Motoya, M. Kasahara, and I. Nakanishi, Automatic processing system for microearthquake network of Hokkaido University, Programme and Abstracts, The Seismological Society of Japan, April 1986, 1-287, 1986 (in Japanese).

TAKANAMI, T., Three-dimensional seismic structure of the crust and upper mantle beneath the orogenic belts in the southern Hokkaido, Japan, J. Phys. Earth, 30, 87-104, 1982.

TrøsтHEIM, D., Autoregressive representation of seismic P-wave signals with an application to the problem of short-period discriminants, Geophys. J. R. Astron. Soc., 43, 269-291, 1975.

Yokota, T., S. Zhou, M. Mizoue, and I. Nakamura, An automatic measurement of arrival time of seismic waves and its application to an on-line processing system, Bull. Earthq. Res. Inst., 55, 449-484, 1981 (in Japanese with English abstract).

\section{APPENDIX}

1. Review of Least Squares Estimation by Householder Transformation Using the matrix $X$ and the vectors $y, a$, and $e$ defined as

$$
\boldsymbol{X}=\left[\begin{array}{llll}
x_{0} & x_{-1} & \cdots & x_{-\boldsymbol{K}} \\
x_{1} & x_{0} & \cdots & x_{2-K} \\
\vdots & \vdots & \vdots: \vdots & \vdots \\
x_{N-1} & x_{N-2} & \cdots & x_{N-K}
\end{array}\right], \quad \boldsymbol{y}=\left[\begin{array}{c}
x_{1} \\
x_{2} \\
\vdots \\
x_{N}
\end{array}\right], \quad \boldsymbol{a}=\left[\begin{array}{l}
a_{1} \\
a_{2} \\
\vdots \\
a_{K}
\end{array}\right], \quad \boldsymbol{e}=\left[\begin{array}{c}
e_{1} \\
e_{2} \\
\vdots \\
e_{N}
\end{array}\right],
$$

Eq. (1) is expressed as

$$
y=X a+e .
$$

It is well known that the least squares solution is given as the solution to the normal equation,

$$
X^{\prime} X a=X^{\prime} y
$$

where $\boldsymbol{X}^{\prime}$ is a transposed matrix of $\boldsymbol{X}$. However, the direct solution of the normal equation is not numerically quite efficient. The so-called square root algorithm which first orthogonalizes the column vectors of $\boldsymbol{X}$ and then solves the resultant equation is known to be superior to the normal equation approach in both numerical accuracy and manipulability in relation to comparison of models and addition of new observations (GoluB, 1965). For any $N \times N$ orthogonal matrix $U$, we have

$$
\|\varepsilon\|^{2}=\|y-X a\|=\|U y-U X a\|^{2} .
$$


Therefore the least squares estimate of $\boldsymbol{a}$ is obtained by minimizing $\|\boldsymbol{U y}-\boldsymbol{U X \boldsymbol { X }}\|^{2}$. $U$ can be chosen so that $\boldsymbol{U X}$ becomes a convenient form for subsequent computations.

Here let us define a $N \times(k+1)$ matrix $Z$ by

$$
Z=[X ; y] \text {. }
$$

Then by the Householder transformation shown in Appendix 2, we obtain a triangular form

$$
\boldsymbol{U} \boldsymbol{Z}=\boldsymbol{S}=\left[\begin{array}{lll}
s_{11} & \cdots & s_{1, K+1} \\
& \cdots & \vdots \\
0 & & \vdots \\
& & s_{K+1, K+1}
\end{array}\right]
$$

For this $U$, we have

$$
\|\boldsymbol{U} \boldsymbol{y}-\boldsymbol{U} \boldsymbol{X} \boldsymbol{a}\|^{2}=\left\|\left[\begin{array}{l}
s_{1, K+1} \\
\\
s_{K, K+1}
\end{array}\right]-\left[\begin{array}{ccc}
s_{11} & \cdots & s_{1 K} \\
& \cdots & \vdots \\
& 0 & \vdots \\
& & s_{K K}
\end{array}\right]\left[\begin{array}{l}
a_{1} \\
\vdots \\
a_{K}
\end{array}\right]\right\|^{2}+s_{K+1, K+1}^{2} .
$$

Then it can be seen that the minimum value of $\|\boldsymbol{U} \boldsymbol{y}-\boldsymbol{U X \boldsymbol { X }}\|^{2}$ is $s_{K+1, K+1}^{2}$ which is attained when the first term of the right-hand side is zero. Further, it can be shown that for each $k \leqq K$, the least squares estimates of the coefficients of the $k$ th order autoregressive model

$$
x_{n}=\sum_{i=1}^{k} a_{i} x_{n-i}+\varepsilon_{n}
$$

are obtained by solving the linear equation

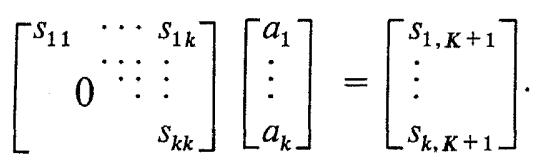

The estimate of the variance of the $k$-th order model, $\sigma^{2}$ is given by

$$
\begin{aligned}
\sigma_{k}^{2} & =\frac{1}{N}\|\varepsilon\|^{2} \\
& =\frac{1}{N}\left(s_{k+1, K+1}^{2}+\cdots+s_{K+1, K+1}^{2}\right) .
\end{aligned}
$$

It should be noted that, in this method, we can get $\sigma^{2}$ and hence AIC without computing the autoregressive coefficient. This property is especially suited to our procedure, since only the AIC values of the various models are necessary.

\section{Householder Transformation}

We will show that any $n \times k$ matrix $\boldsymbol{X}$ can be transformed to an upper 
triangular form by an appropriate orthogonal transformation. As an example of the construction of this transformation, we show a Householder transformation. Given any unit vector $\boldsymbol{w}, U=\mathbb{I}-2 \boldsymbol{w} \boldsymbol{w}^{t}$ defines an orthogonal transformation (mirror transformation) since $U U^{t}=U^{t} U=\mathbb{I}$. Further it can be shown that if $\boldsymbol{a}$ and $\boldsymbol{b}$ are any vectors such that $\|\boldsymbol{a}\|=\|\boldsymbol{b}\|$, then the mirror transformation defined by

$$
w=\frac{a-b}{\|a-b\|}
$$

transforms $\boldsymbol{a}$ to $\boldsymbol{b}$, namely $\boldsymbol{U} \boldsymbol{a}=\boldsymbol{b}$. Therefore for the $n \times k$ matrix $\boldsymbol{X}=\left(x_{i j}\right)$, if we define $a_{1}=\left(x_{11}, x_{21}, \cdots, x_{n 1}\right)^{t}$ and $b_{1}=\left(x_{11}^{(1)}, 0, \cdots, 0\right)^{t}$ with $\left(x_{11}^{(1)}\right)^{2}=\sum_{i=1}^{n} x_{i 1}^{2}$, then the mirror transformation $U_{1} a_{1}$ defined above satisfies $U_{1}=b_{1}$, namely $U_{1} X$ becomes the following form

$$
U_{1} \boldsymbol{X}=\left[\begin{array}{llll}
x_{11}^{(1)} & x_{12}^{(1)} & \cdots & x_{1 k}^{(1)} \\
0 & x_{22}^{(1)} & \cdots & x_{2 k}^{(1)} \\
\vdots & \vdots & & \vdots \\
0 & x_{n 2}^{(1)} & \cdots & x_{n 2}^{(1)}
\end{array}\right]
$$

Similarly by defining $\boldsymbol{U}_{2}$ with $\boldsymbol{a}_{2}=\left(x_{12}^{(1)}, x_{22}^{(1)}, \cdots, x_{n 2}^{(1)}\right)^{t}, b_{2}=\left(x_{12}^{(1)}, x_{22}^{(2)}, 0, \cdots, 0\right)^{t}$, $\left\|a_{2}\right\|=\left\|b_{2}\right\|$, we obtain $U_{2} U_{1} X=\left(b_{1}, b_{2}, *, \cdots, *\right)$. Repeating this transformation for $k$ times, $U_{k} \cdots U_{1} X$ becomes an upper triangular form. 